\title{
Early Detection of Atrial Fibrillation-Atrial Flutter Using Remote Patient Monitoring
}

\author{
Sandy Joung ${ }^{\mathrm{a}}$, Eldin Dzubur ${ }^{\mathrm{b}}$, Irene van den Broek ${ }^{\mathrm{a}, \mathrm{c}}$, Aubrey Love ${ }^{\mathrm{a}}$, Lori Martinez-Rubio ${ }^{\mathrm{a}}$, \\ Mayra Lopez ${ }^{\mathrm{b}}$, Benjamin Noah ${ }^{\mathrm{b}}$, Shivani Dhawan ${ }^{\mathrm{a}}$, Qin Fu ${ }^{\mathrm{a}, \mathrm{c}}$, Mitra Mastali ${ }^{\mathrm{a}, \mathrm{c}}$, \\ Jennifer E. Van Eyk ${ }^{\mathrm{a}, \mathrm{c}}$, Brennan Spiegel ${ }^{\mathrm{b}}$, C. Noel Bairey Merz ${ }^{\mathrm{a}}, \mathrm{d}$, Chrisandra Shufelt ${ }^{\mathrm{a}}$
}

\begin{abstract}
Remote patient monitoring (RPM), patient-reported outcomes (PROs), and biochemical biomarker monitoring (Mitra ${ }^{\circledR}$ devices) may be useful for early detection of major adverse cardiac events (MACE). This case report presents a patient enrolled in a precision medicine study in which RPM detected the presence of atrial fibrillation-atrial flutter (AFib-Flutter), facilitating prompt treatment. A 64-year-old male with a history of ischemic heart disease (IHD) initiated his AliveCor Kardia after noting angina which reported "possible atrial fibrillation". Upon evaluation, AFib-Flutter was confirmed by 12-lead ECG and successfully treated. RPM was recorded continuously through (Fitbit Charge 2), weekly single-channel electrocardiogram rhythm stripe (AliveCor Kardia), PROs through weekly questionnaires, and Mitra ${ }^{\circledR}$ devices through monthly fingerpricks. The case report highlights a successful case of detecting AFib-Flutter, expediting treatment and preventing MACE. Precision medicine using RPM may be useful for detecting AFib-Flutter and improving IHD outcomes. Further research is needed.
\end{abstract}

Keywords: Remote patient monitoring; Precision medicine; Wearable biosensors

\section{Introduction}

Cardiovascular disease (CVD) is the leading cause of mortality, morbidity, and disability in women and men worldwide [1]. Ischemic heart disease (IHD) is the most common cause of death in the USA, affecting 16.8 million individuals [2].

Manuscript submitted August 23, 2018, accepted September 20, 2018

aBarbra Streisand Women's Heart Center, Cedars-Sinai Smidt Heart Institute, Cedars-Sinai Medical Center, Los Angeles, CA, USA

${ }^{b}$ Cedars-Sinai Center for Outcomes Research and Education (CS-CORE), Los Angeles, CA, USA

${ }^{\mathrm{c}}$ Advanced Clinical Biosystems Research Institute, Cedars-Sinai Smidt Heart Institute, Cedars-Sinai Medical Center, Los Angeles, CA, USA

${ }^{\mathrm{d} C}$ Corresponding Author: C. Noel Bairey Merz, 127 S. San Vicente Blvd, Suite A3206, Los Angeles, CA 90048, USA. Email: Noel.BaireyMerz@cshs.org

doi: https://doi.org/10.14740/jmc3151w
Major adverse cardiac events (MACE), such as myocardial infarction, stroke, all-cause mortality, and hospitalizations for arrhythmia, angina, and/or heart failure may occur without warning. In addition, treatment of MACE is costly, which can be a burden for minority populations and those uninsured as there are disproportionally higher rates of MACE among ethnic minority populations due to IHD [3].

By implementing precision medicine through remote patient monitoring (RPM), patient-reported outcomes (PROs), and home biochemical biomarker monitoring (Mitra ${ }^{\circledR}$ devices), clinicians and researchers may improve risk prediction of MACE compared to current risk models. This may also provide the opportunity to improve outcomes by earlier use of pharmaceutical-, procedure-, or device-based therapies [4]. This case report presents how RPM provided an early treatment opportunity by detecting the presence of atrial fibrillation-atrial flutter (AFib-Flutter).

This patient had been enrolled in the "Early Prediction of Major Adverse Cardiovascular Event Surrogates Using Remote Monitoring with Biosensors, Biomarkers, and PatientReported Outcomes" for 90 days. This study was designed to evaluate 200 IHD patients in a prospective design to determine early indicators that may predict MACE by measuring the correlations between RPM, PROs and established MACE biomarker surrogates. During this trial, patients wore wearable biosensors, completed patient reported outcome measures, and obtained blood samples using home-based blood biomarker kits.

\section{Remote patient monitoring wearable biosensors}

RPM data were obtained by continuous use of a wrist-worn Fitbit Charge 2 (Fitbit, San Francisco, CA), and weekly recordings of a standalone electrocardiogram (ECG) by AliveCor Kardia (AliveCor, Mountain View, CA). The Fitbit Charge 2 is a consumer device with an embedded tri-axial accelerometer and a photoplethysmography sensor to track activity and heart rate, respectively. The AliveCor Kardia features a single-channel ECG rhythm strip that detects the presence of atrial fibrillation (AFib) and normal sinus rhythm. The AliveCor Kardia simultaneously records the pulse generated by patients after placing their two index fingers on the electrodes for $30 \mathrm{~s}$ and a voice recording of symptoms experienced. 


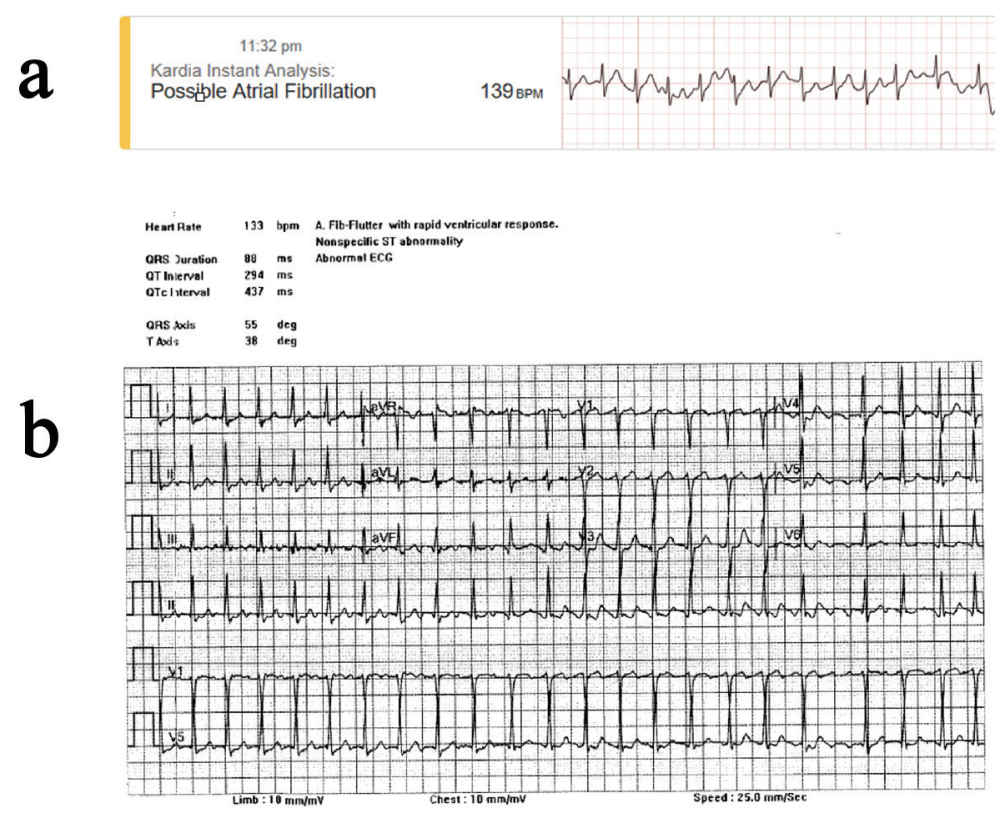

Figure 1. EKG data indicating abnormal arrhythmia as classified by ambulatory and standard EKG. (a) AliveCor Kardia Mobile Device, ambulatory (11:23 PM). (b) 12-lead EKG, hospitalized (12:26 AM).

\section{Patient reported outcomes}

PROs were collected using short-form subscales of the PROMIS $^{\circledR}$ questionnaire to assess depression, emotional distress/anxiety, fatigue, physical function, sleep disturbance, social isolation, global mental health, and global physical health each week [5]. The 7-item Seattle Angina Questionnaire (SAQ-7) assessed physical limitation, angina frequency, quality of life (QOL), and an angina summary score every four weeks [6]. The 12-item Kansas City Cardiomyopathy Questionnaire (KCCQ-12) assessed physical limitation, symptom frequency, QOL, social limitation, and a cardiomyopathy summary score biweekly [7].

\section{Biochemical biomarker monitoring (Mitra ${ }^{\circledR}$ microsam- pling device)}

The Mitra ${ }^{\circledR}$ microsampling device was used to collect blood from a self-administered fingerprick using absorptive sampling tips at four time points: Day 0, Day 30, Day 60, and Day 90. Day 0 and Day 90 samples were collected on site (four replicates), whereas Day 30 and Day 60 samples were collected at-home (two replicates) and mailed to and stored in the laboratory at $-80{ }^{\circ} \mathrm{C}$ until analysis. The target biomarkers (including nine apolipoproteins (apo): apoA-I, apoA-II, apoAIV, apoB, apoC-I, apoC-II, apoC-III, apoE, and apoJ) were extracted from the Mitra ${ }^{\circledR}$ devices $(n=2$ for each time point) using an automated mass spectrometry digestion protocol [8]. Liquid chromatography coupled to tandem mass spectrometry (6500 triple quadrupole LC-MS/MS, Sciex) was used for protein quantification using stable-isotope labeled peptides (New England Peptides) as internal standards. Samples from four other participants, randomly selected in the trial, were used to perform intra- and inter-individual comparisons.

\section{Case Report}

A 64-year-old Caucasian male with a past medical history of percutaneous transluminal coronary angioplasty of the right posterolateral branch in 2016, paroxysmal AFib, hypertension, dyslipidemia and human immunodeficiency virus on antiretroviral treatment was enrolled in a 3-month observational cohort study assessing the value of continuous risk assessment identified by RPM, PROs, and Mitra ${ }^{\circledR}$ devices. The patient had no known family history of heart disease or diabetes and no history of smoking, alcohol or drugs.

Following feelings of angina and palpitations, using the RPM, the patient completed his initial ECG rhythm stripe assessment using his AliveCor Kardia (Fig. 1a). Patient completed a second ECG rhythm stripe assessment 3 min later, voicing into his device "I'm feeling as if I have ... some pressure on my chest". A combination of his initial symptoms, detection of "possible AFib", and elevated heart rate of 139 beats per minute (bpm) on his AliveCor Kardia, led him to call the paramedics and he was taken by ambulance to the emergency department (ED). An EKG conducted upon arrival showed a heart rate of $133 \mathrm{bpm}$, AFib-Flutter with rapid ventricular response and nonspecific ST abnormality (Fig. 1b). Shortly after this initial evaluation, the patient was admitted to the hospital with the diagnosis of AFib-Flutter with rapid ventricular heart rate and chest pain.

Upon admission, cardiac troponin I was negative (reference range: $\leq 0.30 \mathrm{ng} / \mathrm{mL}$ ) and Pro-B-type natriuretic peptide (Pro-BNP) was elevated at $240 \mathrm{pg} / \mathrm{mL}$ (reference range: 0 - 


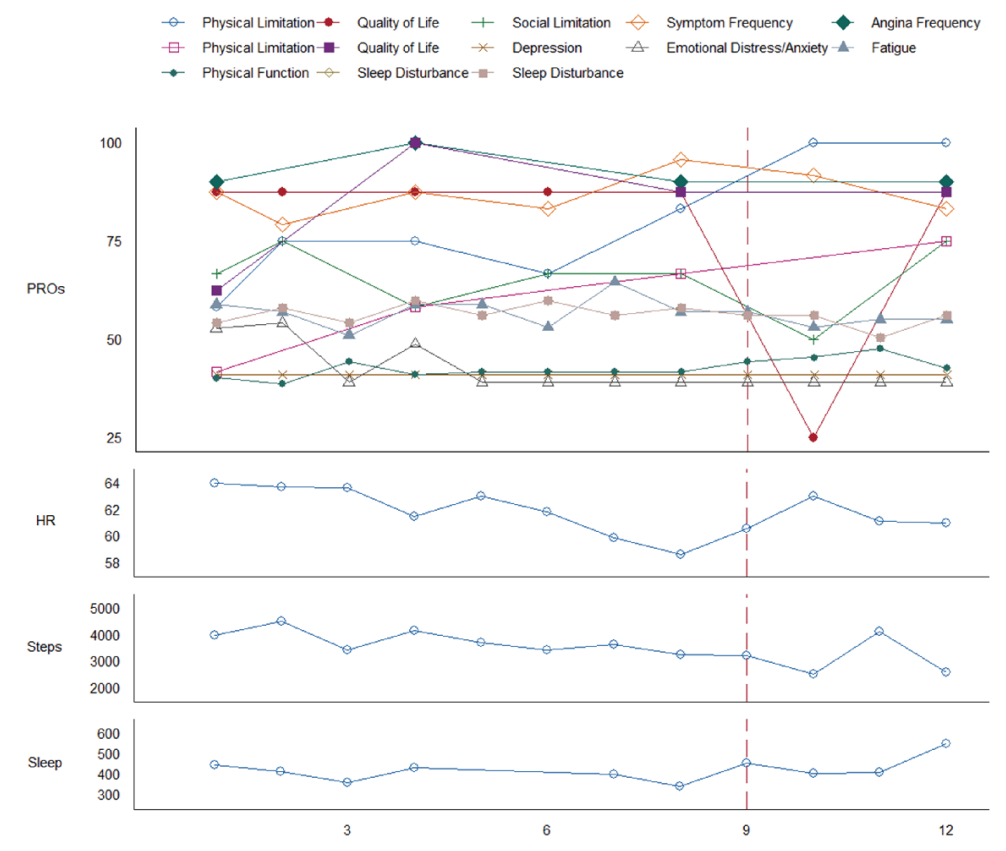

Figure 2. Patient-reported outcomes (PROs) and remote patient monitoring (RPM) measured over 12 weeks. X-axis represents time in weeks. Solid box in legend indicates Kansas City Cardiomyopathy Questionnaire (KCCQ); dotted box in legend indicates Seattle Angina Questionnaire (SAQ). KCCQ and SAQ measures are coded such that low scores indicate poorer outcome, all other measures are coded such that low scores indicate less of a construct. Sleep is in units of minutes per day. A vertical dashed line marks confirmed AFib-Flutter with subsequent hospitalization.

$123 \mathrm{pg} / \mathrm{mL})$. All other labs were within normal range including white blood count of $4.9 \times 10^{3} / \mu \mathrm{L}$ (reference range: $4.8-10.8$ $\times 10^{3} / \mu \mathrm{L}$ ), hemoglobin $13.1 \mathrm{~g} / \mathrm{dL}$ (reference range: $14.2-18.0$ $\mathrm{g} / \mathrm{dL}$ ), hematocrit of $39.1 \%$ (reference range: $42.0-52.0 \%$ ), and platelet count was $108 \times 10^{3} / \mathrm{uL}$ (reference range: $150-450 \times$ $10^{3} / \mathrm{uL}$ ). A chest X-ray showed no signs of acute cardiopulmonary disease and echocardiogram showed normal left ventricular chamber with ejection fraction of $55 \%$, mild left ventricular hypertrophy, and moderate aortic and mitral regurgitation. A second EKG conducted in the ED showed a heart rate of 93 bpm, AFib-Flutter with variable atrioventricular (AV) block, and nonspecific ST abnormality. The patient was monitored on telemetry and was started on anticoagulation therapy with enoxaparin and then managed with calcium channel blocker (diltiazem hydrochloride), converting to sinus rhythm within $12 \mathrm{~h}$ from admission. Due to the rapid improvement in condition, the patient was discharged after 1 day.

The patient's discharge medications included antiplatelet medications (clopidogrel, $75 \mathrm{mg}$ and aspirin $81 \mathrm{mg}$ ), statin (atorvastatin $80 \mathrm{mg}$ ), beta blocker (carvedilol $3.125 \mathrm{mg}$ ), alpha blocker (tamsulosin hydrochloride $0.4 \mathrm{mg}$ ), angiotensin II receptor blocker (valsartan $80 \mathrm{mg}$ ), and his antiretroviral medications (abacavir). Although there was an indication for anticoagulation treatment through the $\mathrm{CHA}_{2} \mathrm{DS}_{2}-\mathrm{VAS}_{\mathrm{C}}$ score for AFib stroke risk [9], the patient declined oral anticoagulation as he wanted to discuss any changes to his treatment with his primary cardiologist. No new medications were added upon discharge. The patient completed a final ECG rhythm stripe on his AliveCor Kardia (92.3\% adherence) post discharge from the hospital which showed normal rhythm and a heart rate of
$70 \mathrm{bpm}$.

The patient's Fitbit reported continuous valid wear time across 90 study days (100\%), with a daily average of $18.9 \mathrm{~h}$ $(\mathrm{SD}=4.8)$ of sedentary time defined as intensity less than 1.5 metabolic equivalents (METs), $3.7 \mathrm{~h}(\mathrm{SD}=3.5)$ of sleep, $1.4 \mathrm{~h}$ $(\mathrm{SD}=1.3)$ of light activity $(3.0>\mathrm{METs}>1.5)$, and $0.1 \mathrm{~h}(\mathrm{SD}$ $=0.1)$ of moderate-vigorous physical activity $($ METs $>3.0)$ [10]. The Fitbit also remotely recorded that the patient slept for $7 \mathrm{~h}$ and $37 \mathrm{~min}$ the night before hospitalizaton, $2 \mathrm{~h}$ the night of hospitalization, and $13 \mathrm{~h}$ after discharge. The patient recorded approximately 2,400 steps the day prior to hospitalization, classified as average in special populations with chronic illness; additional Fitbit activity and heart rate data leading up to hospitalization (Fig. 2) [11]. PROs (100\% response rate) indicated a substantial decline in self-reported QOL immediately following the hospitalization event. Despite the patient's subjective report regarding improvement in physical limitation throughout the study, the object measurement of step count data showed no difference (Fig. 2). There were no significant differences regarding the remaining PROs throughout the duration of the study.

The patient completed his Mitra ${ }^{\circledR}$ devices at all four designated time points (100\%). Apolipoprotein measurements from the dried Mitra ${ }^{\circledR}$ tips were evaluated intra-individually (four points) and inter-individually (four randomly selected patients) (Fig. 3). Apolipoproteins are determinants of atherosclerotic risk and also clinically established biomarkers for cardiovascular risk prediction [12]. For the nine measured apolipoproteins, only the signal for apoC-II at baseline and apoA-IV at 30 days were outside $\mathrm{a} \pm 20 \%$ deviation from the individual's 


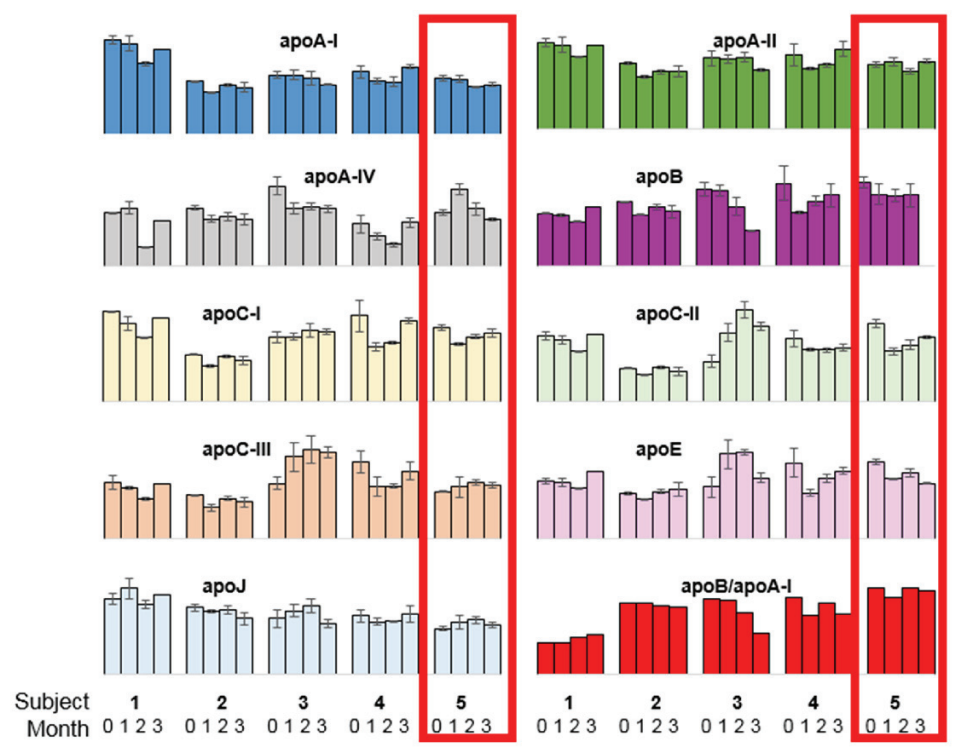

Figure 3. Overview of intra- and inter-individual $(n=5)$ biomarker measurements for nine apolipoproteins and the ratio of apolipoprotein B to apolipoprotein A-I. The five clusters of columns represent five different subjects: 1) No events; 2) Confirmed AFibFlutter (Day 49), not admitted; 3) No events; 4) No events; 5) Confirmed AFib-Flutter (Day 64), admitted (case patient, outlined in red). The four bars within each cluster represent the four sampling time points obtained by Mitra ${ }^{\circledR}$ devices (baseline, 1 month, 2 months, and 3 months). Error bars indicate the standard deviation between duplicate measurements (two tips per time point).

mean. Otherwise, all signals of all nine apolipoproteins were within $\pm 20 \%$ deviation from the overall mean. The ratio of apoB/apoA-I is an established marker surrogate for the traditional lipid panel [12]. When comparing this ratio, the largest inter-individual variability was observed with this patient, + $30.4 \%$ higher than all other individuals. The observed higher ratio apoB/apoA-I ratio is important as it has been proposed as clinically better marker for cardiovascular risk prediction than current lipid (TC/HD) tests $[13,14]$. Nonetheless, intraindividual changes were minor (between $-8.4 \%$ to $4.5 \%$ deviation from the individual's mean). Further analysis of the entire patient cohort $(n=200)$ is required to draw any conclusions on the predictive value of any of the measured biomarkers and their ratios.

From this precision medicine study, the patient appreciated the ability to track his activity, heart rate, ECG rhythm stripe, and record his symptoms in real time. He realized how useful his data were towards self-assessing potentially harmful symptoms and immediately addressing them by calling emergency medical services. Upon exiting the trial, the patient purchased his own RPM wearable biosensors to independently monitor his heart rate and he continued to monitor for arrhythmias through his AliveCor Kardia.

\section{Discussion}

This case highlights the importance of predicting AFib-Flutter using remote monitoring in IHD patients. Through RPM that detected an arrhythmia, this patient sought medical care when he otherwise may have not. Prior to the event, self-reported PROs showed decreased QOL which has been shown in prior studies as an important determinant for further risk. The use of PROs to capture quality-of-life factors such as functional status, depression and anxiety, and pain intensity in real-time may be useful in improving clinical outcomes. Novel to this report is the use of a self-administered finger stick Mitra ${ }^{\circledR}$ microsampling device allowing for the collection of novel biochemical biomarkers, such as apolipoproteins, which may be additive to the RPM data in assessing personalized cardiac risk.

By managing care at earlier stages in IHD, RPM may prevent the need for a costly subsequent therapy and unplanned re-hospitalizations if involving AFib-Flutter. Clinical management and therapy provided in person remains standard of care, however, the use of RPM to conduct remote follow-up and the ability to transfer physiological data such as blood pressure and electrocardiographic directly to the health care provider may alleviate the burden of geographic or funding barriers [15]. Additionally, RPM may also enable better management of IHD risk factors by monitoring populations that are disproportionately impacted by race, ethnicity, socioeconomic status, which accounts for up to $55 \%$ of uninsured [3]. Implementation of consumer-grade wearables in the health system remains a challenge while the adoption of wearable technology favors affluent, young, and relatively healthy individuals [16]. One study noted a potential barrier to RPMs is that patients at highest risk are the least likely to data share when provided with an option to import data from their trackers to an electronic health record [17]. It was unclear as to whether the low uptake was due to the lack of awareness with the patient portal, short monitoring duration, patient trust, or feasibility of use. Nevertheless, other studies have revealed that older adults, including those with chronic disease, are open to using RPM [18]. This precision medicine study observed patient adherence rates up 
to $90.0 \%$ in Fitbit use, demonstrating that RPM interventions may be successful when the devices are free or inexpensive and easy to use [19].

\section{Conclusions}

The case study further elucidates how precision medicine by utilizing changes in RPM, PRO measure scores, and biomarkers obtained from the Mitra ${ }^{\circledR}$ devices has the potential to impact health-related outcomes beyond current established guidelines and predict MACE. RPM devices may also provide timely intervention triggers by allowing researchers and clinicians to assess continuous data collected between office visits. RPM can address the disparities that exist in healthcare by providing a greater range of physiological parameters to more accurately assess and optimize treatments. Further research conducted in RPM, PROs, and Mitra ${ }^{\circledR}$ devices may be useful in evaluating known risk factors and leveraging cost effective strategies in real time, allowing for action to improve health.

\section{Grant Support}

This work was supported by The California State Government: California Initiative to Advance Precision Medicine, San Francisco, California, National Center for Advancing Translational Sciences Grant UL1TR000124 and UL1TR000064, The Barbra Streisand Women's Cardiovascular Research and Education Program, Cedars-Sinai Medical Center, Los Angeles, California, The Society for Women's Health Research (SWHR), Washington, D.C., The Linda Joy Pollin Women's Heart Health Program, and the Erika J. Glazer Women's Heart Health Project, Cedars-Sinai Medical Center, Los Angeles, California, The Cedars-Sinai Center for Outcomes Research and Education (CS-CORE), Cedars-Sinai Medical Center, Los Angeles, California, The Division of Informatics, Cedars-Sinai Medical Center, Los Angeles, California, The Advanced Clinical Biosystems Research Institute, Cedars-Sinai Smidt Heart Institute, Cedars-Sinai Medical Center, Los Angeles, California and supported by in-kind contributions of our industrial partners Neoteryx, Beckman Coulter, Sciex, Thermo Fisher Scientific Inc., and Cambridge Isotope Labs.

\section{Disclosures}

Dr. C. Noel Bairey Merz reports personal honoraria and consulting from ACRWH (NIH Advisory Council), NIHCASE (grant review study section), Springer International (book honorarium), Decision Support in Medicine LLC (book honorarium), and NHLBI Research Triangle Institute (RTI) International, as well as personal research grants for WISE HFpEF (Sponsor: Cedars-Sinai Medical Center, NCT02582021), RWISE (Sponsor: Cedars-Sinai Medical Center, NCT01342029), FAMRI (Sponsor: University of California, San Francisco, NCT01639235), WARRIOR Trial (Sponsor: University of Florida, NCT03417388), and Califor- nia Institute for Precision Medicine (Sponsor: Cedars-Sinai Medical Center, NCT03064360). Dr. Jennifer Van Eyk has a sponsored research agreement with Neoteryx, the company that develops the Mitra ${ }^{\circledR}$ device for volumetric absorptive microsampling of dried blood samples. This project was funded by the State. The contents do not reflect the official views or policies of the State of California.

\section{References}

1. Studzinski K, Tomasik T, Krzyszton J, Jozwiak J, Windak A. Effect of using cardiovascular risk scoring in routine risk assessment in primary prevention of cardiovascular disease: protocol for an overview of systematic reviews. BMJ Open. 2017;7(3):e014206.

2. Cardiovascular disease: a costly burden for america projections through 2035. American Heart Association; 2017. Available from: https://healthmetrics.heart.org/ wp-content/uploads/2017/10/Cardiovascular-Disease-ACostly-Burden.pdf.

3. Leigh JA, Alvarez M, Rodriguez CJ. Ethnic minorities and coronary heart disease: an update and future directions. Curr Atheroscler Rep. 2016;18(2):9.

4. Hlatky MA, Greenland P, Arnett DK, Ballantyne CM, Criqui MH, Elkind MS, Go AS, et al. Criteria for evaluation of novel markers of cardiovascular risk: a scientific statement from the American Heart Association. Circulation. 2009;119(17):2408-2416.

5. Bevans M, Ross A, Cella D. Patient-Reported Outcomes Measurement Information System (PROMIS): efficient, standardized tools to measure self-reported health and quality of life. Nurs Outlook. 2014;62(5):339-345.

6. Chan PS, Jones PG, Arnold SA, Spertus JA. Development and validation of a short version of the Seattle angina questionnaire. Circ Cardiovasc Qual Outcomes. 2014;7(5):640-647.

7. Spertus JA, Jones PG. Development and validation of a short version of the Kansas City cardiomyopathy questionnaire. Circ Cardiovasc Qual Outcomes. 2015;8(5):469476.

8. van den Broek I, Fu Q, Kushon S, Kowalski MP, Millis K, Percy A, Holewinski RJ, et al. Application of volumetric absorptive microsampling for robust, high-throughput mass spectrometric quantification of circulating protein biomarkers. Clinical Mass Spectrometry. 2017;4-5:2533.

9. Coppens M, Eikelboom JW, Hart RG, Yusuf S, Lip GY, Dorian P, Shestakovska O, et al. The CHA2DS2-VASc score identifies those patients with atrial fibrillation and a CHADS2 score of 1 who are unlikely to benefit from oral anticoagulant therapy. Eur Heart J. 2013;34(3):170-176.

10. Kim J, Tanabe K, Yokoyama N, Zempo H, Kuno S. Objectively measured light-intensity lifestyle activity and sedentary time are independently associated with metabolic syndrome: a cross-sectional study of Japanese adults. Int J Behav Nutr Phys Act. 2013;10:30.

11. Tudor-Locke C, Craig CL, Aoyagi Y, Bell RC, Croteau KA, De Bourdeaudhuij I, Ewald B, et al. How many 
steps/day are enough? For older adults and special populations. Int J Behav Nutr Phys Act. 2011;8:80.

12. van den Broek I, Sobhani K, Van Eyk JE. Advances in quantifying apolipoproteins using LC-MS/MS technology: implications for the clinic. Expert Rev Proteomics. 2017;14(10):869-880.

13. McQueen MJ, Hawken S, Wang X, Ounpuu S, Sniderman A, Probstfield J, Steyn K, et al. Lipids, lipoproteins, and apolipoproteins as risk markers of myocardial infarction in 52 countries (the INTERHEART study): a case-control study. Lancet. 2008;372(9634):224-233.

14. Walldius G, Jungner I, Holme I, Aastveit AH, Kolar W, Steiner E. High apolipoprotein B, low apolipoprotein A-I, and improvement in the prediction of fatal myocardial infarction (AMORIS study): a prospective study. Lancet. 2001;358:2026-2033.

15. Hasan A, Paul V. Telemonitoring in chronic heart failure. Eur Heart J. 2011;32(12):1457-1464.
16. Gualtieri L, Rosenbluth S, Phillips J. Can a free wearable activity tracker change behavior? The impact of trackers on adults in a physician-led wellness group. JMIR Res Protoc. 2016;5(4):e237.

17. Pevnick JM, Fuller G, Duncan R, Spiegel BM. A largescale initiative inviting patients to share personal fitness tracker data with their providers: initial results. PLoS One. 2016;11(11):e0165908.

18. Mercer K, Giangregorio L, Schneider E, Chilana P, Li M, Grindrod K. Acceptance of Commercially Available Wearable Activity Trackers Among Adults Aged Over 50 and With Chronic Illness: A Mixed-Methods Evaluation. JMIR Mhealth Uhealth. 2016;4(1):e7.

19. Speier W, Dzubur E, Zide M, Shufelt C, Joung S, Van Eyk JE, Bairey Merz CN, et al. Evaluating utility and compliance in a patient-based eHealth study using continuoustime heart rate and activity trackers. J Am Med Inform Assoc. 2018. 